Document downloaded from:

http://hdl.handle.net/10251/166819

This paper must be cited as:

Gsell, W.; Molinos, C.; Correcher, C.; Belderbos, S.; Wouters, J.; Junge, S.; Heidenreich, M.... (2020). Characterization of a preclinical PET insert in a 7 tesla MRI scanner: beyond NEMA testing. Physics in Medicine and Biology. 65(24):1-16. https://doi.org/10.1088/1361$6560 / a b a 08 c$

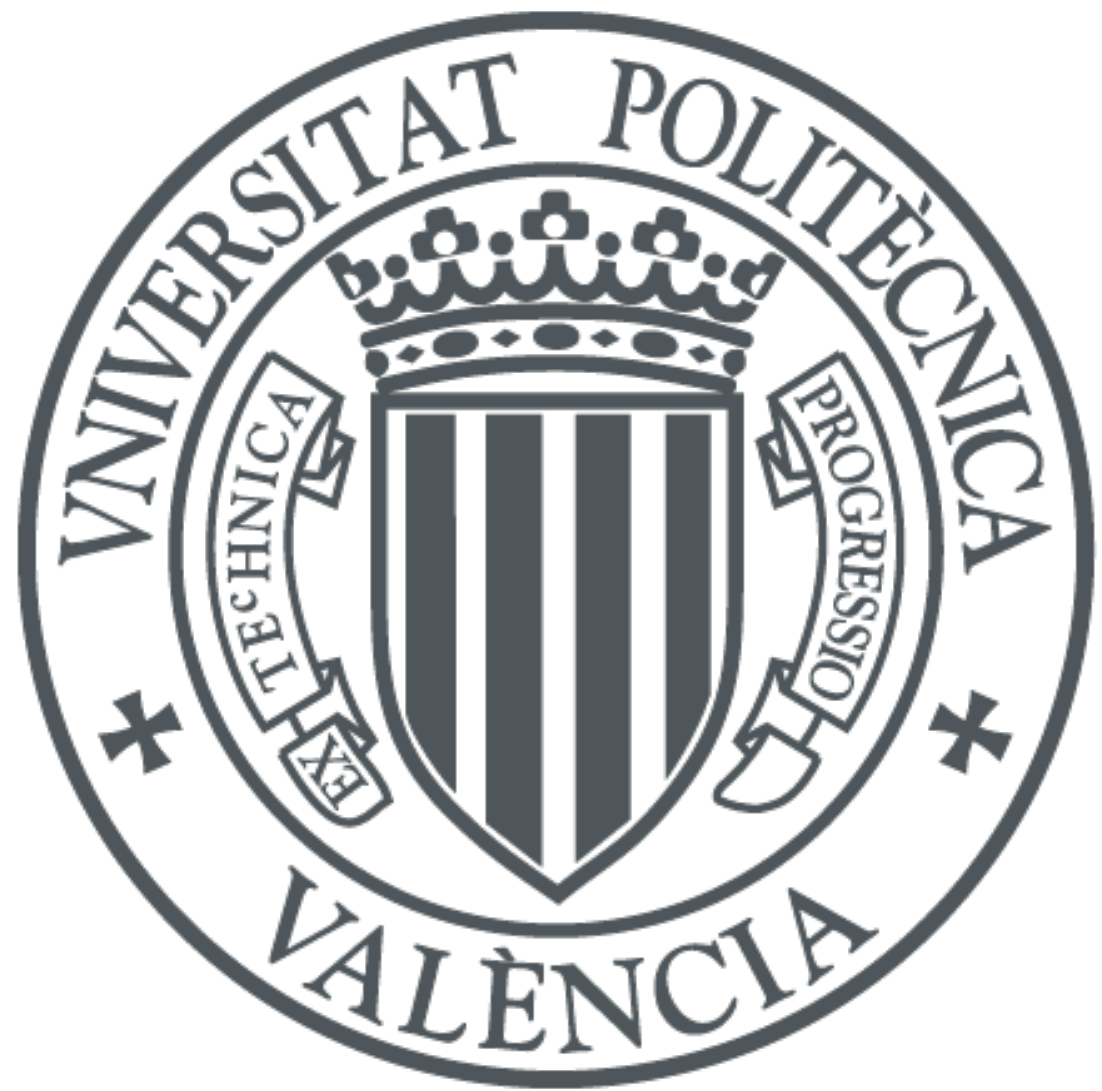

The final publication is available at

https://doi.org/10.1088/1361-6560/aba08c

Copyright IOP Publishing

Additional Information 
ACCEPTED MANUSCRIPT

\section{Characterization of a preclinical PET insert in a 7 Tesla MRI scanner: beyond NEMA testing}

To cite this article before publication: Willy Gsell et al 2020 Phys. Med. Biol. in press https://doi.org/10.1088/1361-6560/aba08c

\section{Manuscript version: Accepted Manuscript}

Accepted Manuscript is "the version of the article accepted for publication including all changes made as a result of the peer review process, and which may also include the addition to the article by IOP Publishing of a header, an article ID, a cover sheet and/or an 'Accepted

Manuscript' watermark, but excluding any other editing, typesetting or other changes made by IOP Publishing and/or its licensors"

This Accepted Manuscript is @ 2020 Institute of Physics and Engineering in Medicine.

During the embargo period (the 12 month period from the publication of the Version of Record of this article), the Accepted Manuscript is fully protected by copyright and cannot be reused or reposted elsewhere.

As the Version of Record of this article is going to be / has been published on a subscription basis, this Accepted Manuscript is available for reuse under a CC BY-NC-ND 3.0 licence after the 12 month embargo period.

After the embargo period, everyone is permitted to use copy and redistribute this article for non-commercial purposes only, provided that they adhere to all the terms of the licence https://creativecommons.org/licences/by-nc-nd/3.0

Although reasonable endeavours have been taken to obtain all necessary permissions from third parties to include their copyrighted content within this article, their full citation and copyright line may not be present in this Accepted Manuscript version. Before using any content from this article, please refer to the Version of Record on IOPscience once published for full citation and copyright details, as permissions will likely be required. All third party content is fully copyright protected, unless specifically stated otherwise in the figure caption in the Version of Record.

View the article online for updates and enhancements. 


\title{
Characterization of a preclinical PET insert in a 7 Tesla MRI scanner: beyond NEMA testing
}

\author{
Willy Gsell ${ }^{1}$, Cesar Molinos ${ }^{2}$, Carlos Correcher ${ }^{2}$, Sarah Belderbos ${ }^{1}$, Jens Wouters ${ }^{3}$, \\ Sven Junge ${ }^{2}$, Michael Heidenreich ${ }^{2}$, Greetje Vande Velde ${ }^{1}$, Ahmadreza Rezaei ${ }^{4}$, \\ Johan Nuyts ${ }^{4}$, Christopher Cawthorne ${ }^{3}$, Frederik Cleeren ${ }^{5}$, Lise Nannan ${ }^{1}$, \\ Christophe M. Deroose ${ }^{4}$, Uwe Himmelreich ${ }^{1 \#}$, Antonio J. Gonzalez ${ }^{6}$
}

${ }^{1}$ Biomedical MRI group, Department of Imaging and Pathology, KU Leuven, Leuven, Belgium.

${ }^{2}$ Nuclear Molecular Imaging, Bruker BioSpin Preclinical Imaging Division, Ettlingen, Germany.

${ }^{3}$ Molecular Small Animal Imaging Center (MoSAIC), Department of Imaging and Pathology, KU Leuven, Leuven, Belgium.

${ }^{4}$ Nuclear Medicine and Molecular Imaging, Department of Imaging and Pathology, KU Leuven, Leuven, Belgium.

${ }^{5}$ Radiopharmaceutical research, Department of Pharmaceutical and Pharmacological sciences, KU Leuven, Leuven, Belgium

${ }^{6}$ Instituto de Instrumentación para Imagen Molecular (I3M), Centro Mixto CSIC - Universitat Politècnica de València, Valencia, Spain.

\# Corresponding author:

Pr. Uwe Himmelreich

Biomedical MRI group

Gasthuisberg Campus, O\&N1 building

Herestraat 49, B-3000 Leuven

Tel: +32(0)16330925

e-mail: Uwe.himmelreich@kuleuven.be

Received xxxxxx

Accepted for publication $\mathrm{xxxxxx}$

Published xxxxxx

\begin{abstract}
This study evaluates the performance of the Bruker PET insert combined with a BioSpec 70/30 USR MRI scanner using the manufacturer acceptance protocol and the NEMA NU 4-2008 for small animal positron emission tomographs (PET). The PET insert is made of 3 rings of 8 monolithic LYSO crystals $\left(50 \times 50 \times 10 \mathrm{~mm}^{3}\right)$ coupled to silicon photomultipliers (SiPM) arrays, conferring an axial and transaxial FOV of $15 \mathrm{~cm}$ and $8 \mathrm{~cm}$. The MRI performance was evaluated with and without the insert for the following radiofrequency noise, magnetic field homogeneity and image quality, For the PET performance, we extended the NEMA protocol featuring system sensitivity, count rates, spatial resolution and image quality to homogeneity and accuracy for quantification using several MRI sequences (RARE, FLASH, EPI and UTE). The PET insert does not show any adverse effect on the MRI performances. The MR field homogeneity is well preserved (Diameter Spherical Volume, for $20 \mathrm{~mm}$ of $1.98 \pm 4.78$ without and $-0.96 \pm 5.16 \mathrm{~Hz}$ with the PET insert). The PET insert has no major effect on the radiofrequency field. The SNR measurements also do not show major differences. Image ghosting is well within the manufacturer specifications $(<2.5 \%)$ and no RF noise is visible. Maximum sensitivity of the PET insert is $11.0 \%$ at the center of the FOV even with simultaneous acquisition of EPI and RARE. PET MLEM resolution is $0.87 \mathrm{~mm}$ (FWHM) at $5 \mathrm{~mm}$ off-center of the FOV and $0.97 \mathrm{~mm}$ at $25 \mathrm{~mm}$ radial offset. The peaks for true/noise equivalent count rates are 410/240 and 628/486 kcps for the rat and mouse phantoms, and are reached at 30.34/22.85 and 27.94/22.58 MBq. PET image quality is minimally altered by the different MRI sequences. The Bruker PET insert shows no adverse effect on the MRI performance and demonstrated a high sensitivity, sub-millimeter resolution and good image quality even during simultaneous MRI acquisition.
\end{abstract}

Keywords: Preclinical, PET-insert, MRI, performances, Imaging 


\section{Introduction}

While Positron Emission Tomography (PET) is known for its high sensitivity, it suffers from limited spatial resolution, and lack of anatomical information (Vaquero and Kinahan 2015). To overcome these, PET/computed tomography (CT) hybrid systems were introduced during the 90's (Townsend 2008, Von Schulthess and Schlemmer 2009). These integrated multi-modal imaging approaches have considerably improved the patient throughput (Steinert and Von Schulthess 2002), the precision of the PET signal localization and quantification (Zaidi et al. 2008), but also led to the development of CTbased attenuation correction (Kinahan et al. 1998). Nowadays, this correction approach is considered as the gold standard method over the lengthy PET transmission scan using external radioactive sources. However, while $\mathrm{CT}$ provides valuable information for bone and lung imaging, it is still limited by its poor soft tissue contrast. Moreover, CT uses ionizing radiation and remains mainly a structural imaging modality at least for preclinical applications (Clark and Badea 2014, Nanni and Torigian 2008). This provided the motivation to combine PET and magnetic resonance imaging (MRI). Indeed, besides the original interest to use a magnetic field to improve the PET spatial resolution through reduction of positron range (Hammer et al. 1994), MRI provides high soft tissue contrast, multi-parametric read-outs (MRI-diffusion, perfusion, dynamic contrast enhancement, etc.) and does not add additional exposure to ionizing radiation (Drzezga et al. 2012 Jadvar and Colleti 2014, Vandenberghe and Marsden 2015, Schug et al. 2016) as opposed to CT. While the PET/MRI hybrid concept originated before the PET/CT, it took longer to materialize due to technical difficulties, the complexity to integrate PET within the strong magnetic field and the higher cost. To encompass the cost issue and make use of already installed MRI systems, PET insert designs were proposed (Grant et al. 2017, Benlloch et al. 2018) and became the preferred option by most manufacturers of preclinical MRI equipment (Wehrl et al., 2011, Mannheim et al. 2018). From a technical point of view, the design of a PET insert for preclinical imaging has to overcome several challenges due to the high static magnetic field, the fast switching of the gradient coils and the interaction of the radiofrequency (RF) field with the PET electronics. Preclinical MRI feature comparatively stronger gradients than clinical systems so the potential for adverse interactions is higher. The first concepts of MRIcompatible PET detectors made use of conventional photomultiplier tubes placed outside the magnetic field and read out by means of long optic fibers (Shao et al. 1997, Catana et al. 2006, Raylman et al. 2006, Yamamoto et al. 2010). This approach is no longer pursued because of the limited space inside the MRI, the strong magnetic field (79.4T) used in preclinical scanners and the deterioration of the PET performance (energy and time resolution). These limitations have now been overcome using solid-state detectors such as avalanche photodiodes (APD) (Pichler et al. 2006, Judenhofer et al. 2007, Wehrl et al. 2011, Maramraju et al. 2011) and more recently silicon photomultipliers (SiPM) (Roncali and Cherry 2011, Yamamoto et al. 2012, Kang et al. 2015, Wehner et al. 2015, Gonzalez et al. 2016, Ko et al. 2016, Schug et al. 2016, Omidvari et al. 2017, 2018, Stortz et al. 2018) which are insensitive to strong magnetic fields and therefore offer excellent performance even when inside magnet bores similar to photomultiplier tubes. In this work, we report the performance of a novel preclinical PET insert based on monolithic LYSO crystals and high-density SiPM arrays. The aim of this work is also to investigate the mutual interaction of the MRI and PET sub-systems while operated in simultaneous mode using a comprehensive set of acquisitions adapted from both the NEMA NU 4-2008 standard and the MRI manufacturer acceptance protocols. Furthermore, we propose this as a framework to harmonize the testing of preclinical PET inserts in general.

\section{Methods}

\subsection{System description}

\subsubsection{PET}

The PET insert is made of 3 rings of detector blocks including a monolithic LYSO crystal $\left(50 \times 50 \times 10 \mathrm{~mm}^{3}\right)$, coupled to an array of $12 \times 12$ SiPMs with $3 \times 3 \mathrm{~mm}^{2}$ active area each $(4.2 \mathrm{~mm}$ pitch) and using a projection readout (Gonzalez et al. 2016). PET signals are fed to a data acquisition system with 12-bit analog to digital converters, $250 \mathrm{~ns}$ integration window and a programmable coincidence window of 3,5, 7 and 9 ns. The outer and inner diameters (OD, ID) of the insert are 198 and $114 \mathrm{~mm}$. The system has a transaxial field of view (FOV) of $80 \mathrm{~mm}$ and an axial FOV of $150 \mathrm{~mm}$. The system is temperature stabilized $\left( \pm 0.2{ }^{\circ} \mathrm{C}\right)$ using controlled air cooling in the range of $20-25^{\circ} \mathrm{C}$. The PET rings are surrounded by a toroidal structure made out of thin carbon fiber composites, to avoid radio-frequency interferences, and possible eddy currents by the switching gradient fields (Gonzalez et al. 2019). The PET electronics were shielded from the RF field, without generating eddy currents arising from the switching gradient field, by implementing a cavity made of 3 layers of $200 \mu \mathrm{m}$ overlaying carbon fiber sheets.

\subsubsection{MRI}

The MRI system is a horizontal small animal 7T MRI scanner (BioSpec 70/30, Bruker BioSpin, Ettlingen, Germany) with a bore size of $30 \mathrm{~cm}$ (ID) and equipped with $20 \mathrm{~cm}$ ID actively shielded gradients $(200 \mathrm{mT} / \mathrm{m})$. Two quadrature birdcage transmit/receive radio-frequency (RF) coils have been designed to work with the PET insert and were tested in this study: a mouse quadrature body coil with an ID of $40 \mathrm{~mm}$ (Quad40) and an $86 \mathrm{~mm}$ ID (Quad86) for rat imaging. 


\subsection{MRI performance}

All MRI acquisition parameters are summarized in table 1.

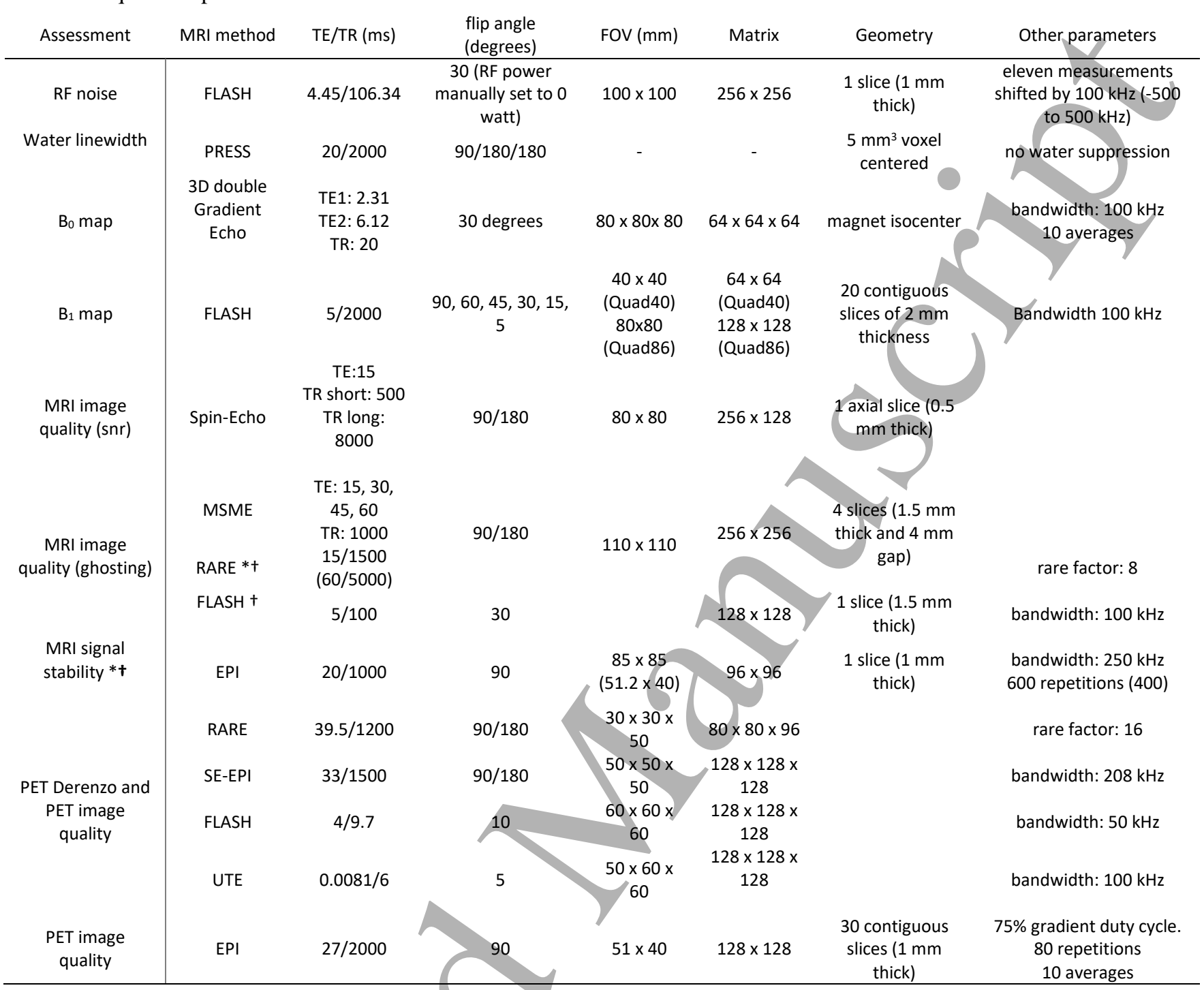

Table 1. MRI acquisition parameters. FLASH: Fast Low Angle SHot, PRESS: Point RESolved Spectroscopy, MSME: MultiSlice Multi-Echo, RARE: Rapid Acquisition with Refocused Echo, SE: Spin-Echo, EPI: Echo Planar Imaging, UTE: Ultra Short Echo Time.* Similar parameters used for PET resolution and PET linearity acquisition $\dagger$.

\subsubsection{Radiofrequency noise}

The leak-through of radiofrequency (RF) noise induced by the presence of the PET insert was qualitatively assessed by acquiring a gradient echo sequence (FLASH) without any RF transmission (RF transmit power set to 0 watt). These measurements were performed with and without the presence of the PET insert. For each measurement, data were visually checked first. Thereafter, we generated a RF noise map (each line corresponding to each frequency range and each column to the binning of the sum of all phase encoding). Each noise map was then normalized to the average value and scaled from 0.5 to 1.5 (see figure. 1 ). 


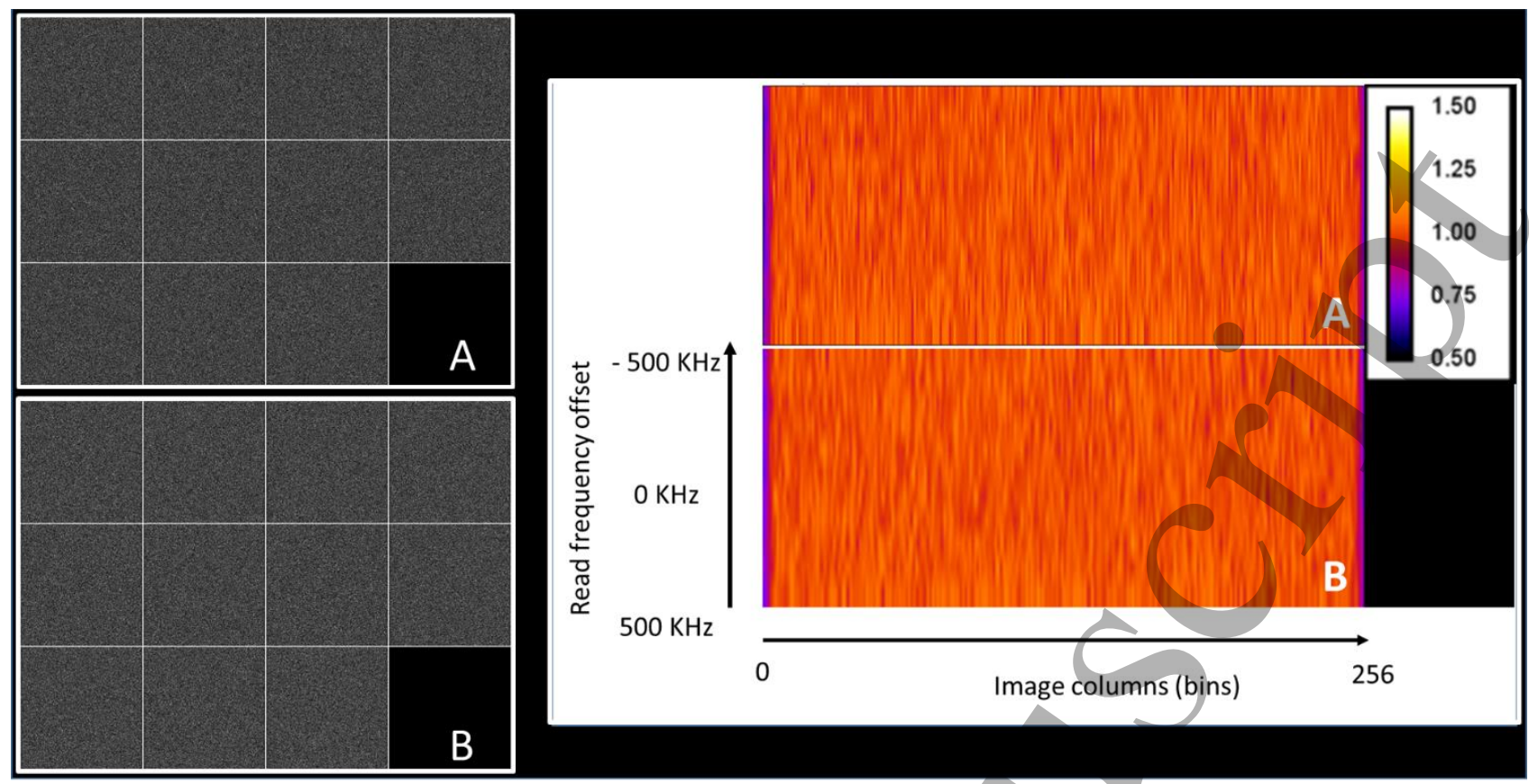

Figure 1. MRI radiofrequency noise in the presence (B) or absence (A) of the PET insert. Each image in the left A and B panels correspond to images acquired at different frequency offset (from -500 to $500 \mathrm{kHz}$ with increment of $100 \mathrm{kHz}$ ) using a FLASH sequence with transmit power set to zero. The right panel shows noise maps in both conditions.

\subsubsection{Linewidth and magnetic field homogeneity}

As a primary assessment, MR data using a Point RESolved Spectroscopy (PRESS) sequence were acquired without the presence of the PET insert using a spherical phantom of 55 mm ID filled with a solution of $\mathrm{CuSO}_{4}(1 \mathrm{~g} / \mathrm{L})$ centered in the MRI FOV. Then, the RF coil was removed and the PET insert was placed. The same acquisition was repeated without moving the phantom and with no pre-adjustment of the center frequency and shims, in order to evaluate the possible effect of the PET insert. Linewidth FWHM and FWTM were determined for both conditions. In a different set/ of experiments, field maps were acquired without the PET insert, with the PET insert but without adjustments (center frequency and shims) and, with the PET insert after pre-adjustments. The parametric field maps were generated by calculating the phase difference between each echo for voxels having an SNR of at least 15 and displayed as the deviation in $\mathrm{Hz}$ or ppm to the center frequency. As depicted in figure 2, spherical regions of interest (DSV) of 20 and $40 \mathrm{~mm}$ in diameter were drawn to assess the respective field homogeneity in each condition.

\subsection{3 $\quad B_{1}$ field mapping}

The radiofrequency field was quantitatively assessed (Balezeau et al. 2011) for the Quad40 and Quad86 coils. For the Quad40 coil, a $50 \mathrm{ml}$ conical centrifuge tube filled $\mathrm{CuSO}_{4}$ solution ( $1 \mathrm{~g} / \mathrm{L}$, known T1 relaxation of $260 \mathrm{~ms}$ ) was used. For the Quad86, we used a cylindric agar phantom $(1 \mathrm{~g} / \mathrm{L} \mathrm{CuSO} 4$,
$10 \mathrm{~g} / \mathrm{L}$ agar dissolved in distilled water) with an internal diameter of $60 \mathrm{~mm}$.

The flip angle maps were obtained under 3 different conditions: PET insert in the FOV and powered ON, PET insert in the FOV but powered OFF and finally with no PET insert in the FOV and for nominal flip angles of 60, 45, 30, 15 and 5 degrees. Normalized pulse magnitude maps were also generated as a mean to compare the different conditions for the efficiency of the radiofrequency accuracy even for low flip angles. Mean and standard deviation of the flip angles and their normalized magnitude were extracted from cylindric volumes of interest of $20 \mathrm{~mm}$ and $60 \mathrm{~mm}$ diameters over a length of $40 \mathrm{~mm}$ for the Quad40 and Quad86, respectively (Figure 3).

\subsubsection{Image quality}

Image quality was assessed for Quad40 and Quad86. At first, all tests were performed without the PET insert. Afterwards, without moving the phantom from the FOV, the PET insert was placed inside the bore of the magnet. All MRI sequences were then re-acquired after re-adjustment of center frequency, shims, transmit power and receiver gain. For the Quad86, the same spherical phantom with $55 \mathrm{~mm}$ ID was centered into the MRI scanner. For the smaller coil (Quad40), a $50 \mathrm{ml}$ conical centrifuge tube filled with the same $\mathrm{CuSO}_{4}$ solution was used. Signal to Noise (SNR), homogeneity and left-right symmetry was assessed using a standard single slice Spin-Echo sequence with a short and a long repetition time. Image ghosting was evaluated using MultiSlice MultiEcho (MSME), rapid acquisition with refocused echoes (RARE) and FLASH 
sequences. Each acquisition was performed in axial, coronal and sagittal orientation using the centrifuge tube phantom. For the SNR, the automatic algorithm provided by the manufacturer was used. It automatically measures and determines the SNR based on a contour finding algorithm for signal and noise regions. Finally, it normalizes the SNR to a volume of one $\mathrm{mm}^{3}\left(\mathrm{SNR} / \mathrm{mm}^{3}\right)$ which is compared with the SNR specification of the QA (Quality Assurance). The initial ROI was then divided into two identical ROIs for left and right, and the symmetry reported as the difference between each side divided by the average mean. As for the ghosting, four ROIs were delineated under Paravision 6.0.1 (ROI 1: signal from the sample, ROI 2: signal and ghost from the sample; ROI 3 and 4: pure noise at the right and left of the image). Ghosting was computed as the difference of signals between the region containing the ghost and the noise, divided by the signal from the sample.

\subsubsection{Signal stability}

Signal stability over time was measured through the use of a single slice EchoPlanar Imaging (EPI) sequence with and without the PET insert using the Quad86 set up and with the $55 \mathrm{~mm}$ in diameter spherical phantom. A spherical region of interest of $55 \mathrm{~mm}$ in diameter was placed on the image corresponding to the first repetition and the time-course of the normalized signal (ROI signal divided by the mean of the ROI signal over time) was plotted. ROI peak to peak divided by mean was reported.

\subsection{PET performance}

\subsubsection{PET module performance (energy resolution, depth of interaction and linearity)}

We investigated the performance of 3 different PET detector blocks (M0, M10 and M20) positioned at 0, 90 and 180 degrees on the first, second and third ring respectively. Data from the linearity assessment were used. In particular, we used the first and last sets of data. The initial activities for the first set were 44.3, 41.3, 38.3 and $35.2 \mathrm{MBq}$ for the case without MRI sequence, FLASH, RARE and EPI. For the last set of data the initial activities were 1.1, 1.0, 0.9 and $0.8 \mathrm{MBq}$, respectively. The energy resolutions for each detector block and case were obtained for a ROI of $2 \times 2 \mathrm{~cm}^{2}$, and calculated as the full width at half maximum (FWHM) divided by the centroid. Depth of interaction (DOI) plots were also obtained for a center ROI in the detector block using the RAW data. The DOI histograms were fitted to a function well suited for the measured profiles especially when crystals are black painted (Gonzalez et al. 2016). As a result, one can obtain information about the DOI resolution, among other parameters.

\subsubsection{PET resolution}

A NEMA compliant sodium-22 (Na-22) small size source, embedded in a 1.0-cm-wide acrylic cube (model MMS09; Eckert \& Ziegler Isotope Products) of $\leq 0.25 \mathrm{~mm}$ in diameter and $0.27 \mathrm{MBq}$ activity, was scanned at radial offsets of 0,5 , $10,15,25$ and $35 \mathrm{~mm}$ at the axial center and at an offset of one quarter the length of the field of view (FOV) from the axial center $(+37.5 \mathrm{~mm})$. All acquisitions were first performed inside the MRI without any MRI sequence and, later, enabling RARE and EPI sequences, with at least $10^{6}$ coincidences (5 min acquisition time). All data were reconstructed using maximum likelihood expectation maximization (MLEM) reconstruction with 36 iterations and an isotropic voxel size of $0.25 \mathrm{~mm}$. The FWHM and full width at tenth of the maximum (FWTM) of the line profile in radial, tangential and axial directions were determined using Amide software (Amide $1.0 .4,31)$ according to the protocol defined by the NEMA NU 4-2008 standard. Images using a Derenzo-like phantom (rod diameters of $0.7,0.8,0.9,1,1.2$ and $1.5 \mathrm{~mm}$ ) were also acquired to study the overall resolution and image quality, simultaneously with different 3D MRI sequences namely 3DRARE, 3D-FLASH, 3D-SE-EPI and 3D-UTE. For this purpose, the phantom was filled with a nominal activity of 30 MBq of $\left[{ }^{18} \mathrm{~F}\right] \mathrm{FDG}$ and placed at the center of the FOV. Static scans were acquired without MRI sequence (20 min), 3DRARE (10 min), 3D-FLASH (14 min), 3D-SE-EPI (14 min) and 3D-UTE (14 min) having an activity of 13.3, 7.7, 6.9, 6.3 and $5.6 \mathrm{MBq}$ at the start of each scan. PET data were corrected for scatter and random events during MLEM reconstruction $(0.25 \mathrm{~mm}$ voxel size, 100 iterations and partial volume correction (PVC) based on the deconvolution of the point spread function).

\subsubsection{PET sensitivity}

Using the same $\mathrm{Na}-22$ point source previously described and according to the NEMA NU4 2008, the sensitivity profile along the axial axis of the FOV was determined in steps of 1 $\mathrm{mm}$ around the center FOV and then by steps of $5 \mathrm{~mm}$ until the edge of the FOV. Three acquisitions of 1 minute were acquired at each position (no MRI sequence running and two MRI sequences, RARE and EPI). Background (with no activity in the PET FOV) was also acquired under the same conditions (without MRI sequence, RARE and EPI).

\subsubsection{NECR and scatter fraction}

Noise Equivalent Count Rates (NECR) were also measured according to the NEMA NU 4-2008 protocol using both the mouse- and rat-sized count-rate phantoms. During both acquisitions, the PET insert was placed inside the MRI scanner with the gradient coil powered ON but without the RF coil inside the PET FOV. Initial activities for each phantom were 126.9 and 46.4 MBq of fluorine-18 (F-18) for the mouse and rat phantoms. Dynamic acquisitions lasting $1 \mathrm{~min}$ for each frame and interleaved with $10 \mathrm{~min}$ gap, were acquired during 19 hours.

\subsubsection{PET image quality}

PET image quality was assessed using the NEMA image quality phantom from which we derived the homogeneity, recovery coefficients, and spill-over ratios according to the 
protocol. The phantom was acquired without MRI sequence, and simultaneously with 3D-SE-EPI, 3D-FLASH, 3D-RARE, 3D-UTE and 2D-EPI at high gradient duty cycles, respectively. All PET data were reconstructed using MLEM, with $0.25 \mathrm{~mm}$ isotropic voxel size and 25 iterations.

\subsubsection{PET linearity and quantification accuracy}

A cylindrical homogenous phantom with $30 \mathrm{~mm}$ ID and 140 mm length was filled with $50.2 \mathrm{MBq}\left[{ }^{18}\right.$ F]FDG. Series of 17 min acquisitions were acquired alternating with different MRI conditions (no sequence, FLASH, RARE and EPI). Acquisitions were run until the activity decayed to $40 \mathrm{kBq}$. Data was reconstructed using MLEM with $0.75 \mathrm{~mm}$ isotropic voxel size and 36 iterations. From these data, the linearity for the different sequences was extracted using a centered cylindrical ROI of $15 \mathrm{~mm}$ in diameter and $45 \mathrm{~mm}$ length. For linear regression, the range for calibration of the scanner ( 1 to $30 \mathrm{MBq}$ ) was used and average errors were calculated for the different conditions (no MRI, FLASH, RARE and EPI) to approximate the quantification accuracy under the different scenarios. The "no MRI" condition was used to approximate the quantification accuracy under the different conditions (FLASH, RARE and EPI).

\subsection{In-vivo imaging}

All animal experiments were approved by the Ethical Committee of the KU Leuven (ECD projects P226/2015, P259/2015 and P060/2018) and were conducted according to the Belgian (Royal Decree of 29 May 2013), Flemish (Decision of the Flemish Government to adapt the Royal Decree of 29 May 2013, 17 February 2017) and European (Directive 2010/63/EU) regulations on the protection of animals used for scientific purposes. In the first application (figure 9A), we acquired rat brain images using $\left[{ }^{18} \mathrm{~F}\right] \mathrm{FDG}$. Shortly, after 12 hours of fasting the rat was anesthetized with $2 \%$ isoflurane in $100 \%$ oxygen. $\left[{ }^{18} \mathrm{~F}\right] \mathrm{FDG}(51 \mathrm{MBq})$ was then intravenously injected and the animal left under anesthesia for 75 min before starting the imaging process. The animal was then placed in an MRI-compatible rat cradle with ear and tooth bars to avoid head motion. The data were acquired using the Quad86 as a transmit coil and a rat brain quadrature shaped surface coil (Bruker Biospin, Ettlingen) as a receiver. A 2D $\mathrm{T}_{2}$ weighted TurboRARE sequence (TE/TR: $33 / 2500 \mathrm{~ms}$, RARE factor: 8, FOV: 35 x $35 \mathrm{~mm}$, matrix: $256 \times 256,15$ slices of 1 $\mathrm{mm}$ thickness and $0.2 \mathrm{~mm}$ gap, 2 averages) was used, combined with a simultaneous 60 min static PET acquisition. PET data were reconstructed using MLEM with $0.5 \mathrm{~mm}$ voxel size and 24 iterations.

For the second animal study (figure $9 \mathrm{~B}$ and C), we used a male Sprague Dawley rat (bodyweight $313 \mathrm{~g}$ ). The animal was not fasted to preserve a good myocardial uptake of $\left[{ }^{18} \mathrm{~F}\right] \mathrm{FDG}$ after an intravenous injection of $37.9 \mathrm{MBq}$ activity. An ECGgated PET acquisition was performed 120 minutes post injection. PET data was reconstructed for 8 cardiac frames using MLEM (0.5 mm voxel size and 24 iterations). Finally, for the third study (figure 9 D), a C57BL6 female mouse was injected with $15.0 \mathrm{MBq}$ of $\left[{ }^{18} \mathrm{~F}\right] \mathrm{NaF}$ and scanned 40 min post injection for $60 \mathrm{~min}$. Data was reconstructed using MLEM (0.25 mm voxel size, 35 iterations and PSF-based PVC). MRI was acquired using a 2 bed positions 3D-TurboRARE (TE/TR: 27/500 ms, RARE factor: 8, FOV: 5030 x $30 \mathrm{~mm}$, matrix: $250 \times 150 \times 100$, respiratory gated, 15 min acquisition per bed position) with the second bed position being simultaneously acquired with the PET sub-system.

\section{Results \\ 3.1 MRI performance}

As shown in figure 1, no radiofrequency noise induced by the PET insert was detected within the investigated $1000 \mathrm{kHz}$ bandwidth. The magnetic field homogeneity was affected by the presence of the PET insert (figure 2) with a shift of the center frequency of $18.87 \mathrm{~Hz}$ and a decrease of the local homogeneity as attested by the increase of the standard deviation of the DSV20 and DSV40. These induced inhomogeneities were all compensated by recalculating the center frequency and applied shims adjustments $(1.98 \pm 4.78$

Without PET Difference (\%)

\begin{tabular}{|c|c|c|c|c|c|c|c|}
\hline \multirow[t]{2}{*}{ Coil } & & 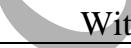 & ET & \multicolumn{2}{|c|}{ Without PET } & \multicolumn{2}{|c|}{ Difference $(\%)$} \\
\hline & & Short TR & Long TR & Short TR & Long TR & Short TR & Long TR \\
\hline \multirow{16}{*}{ Quad 86} & SNR per $\mathrm{mm}^{3}$ & 1198 & 1992 & 1270 & 2032 & & \\
\hline & SNR & 300 & 498 & 318 & 508 & -5.0 & -2.0 \\
\hline & L-R symmetry $(\%)$ & 0.8 & 0.6 & 0.6 & 0.5 & -0.2 & -0.1 \\
\hline & Homogeneity (\%) & 0.8 & 0.9 & 0.7 & 2.7 & -0.1 & 1.8 \\
\hline & Reference power (W) & 1.3 & 1.3 & 1.3 & 1.3 & - & - \\
\hline & Receiver gain (db) & 36 & 12.7 & 36 & 12.7 & - & - \\
\hline & & \multirow{3}{*}{\multicolumn{2}{|c|}{$\begin{array}{c}0.84 \text { (MSME) } \\
1.07 \text { (FLASH) } \\
0.76 \text { (RARE) }\end{array}$}} & \multirow{3}{*}{\multicolumn{2}{|c|}{$\begin{array}{c}1.33 \text { (MSME) } \\
0.82(\mathrm{FLASH}) \\
0.83 \text { (RARE) }\end{array}$}} & \multirow{3}{*}{\multicolumn{2}{|c|}{ Specified $<2.5 \%$}} \\
\hline & Image ghosting $(\%)$ & & & & & & \\
\hline & & & & & & & \\
\hline & SNR per $\mathrm{mm}^{3}$ & 2892 & 5248 & 2936 & 5154 & \multirow{2}{*}{-1.5} & \multirow{2}{*}{1.8} \\
\hline & SNR & 141 & 1312 & 143 & 1288 & & \\
\hline & L-R symmetry (\%) & 0.1 & 0.3 & 0.1 & 0.2 & 0 & -0.1 \\
\hline & Homogeneity (\%) & 0.7 & 0.5 & 0.6 & 0.5 & -0.1 & 0 \\
\hline & Reference power (W) & 0.216 & 0.216 & 0.216 & 0.216 & - & - \\
\hline & Receiver gain $(\mathrm{dB})$ & 64 & 22.6 & 64 & 22.6 & - & - \\
\hline & Image ghosting $(\%)$ & \multicolumn{2}{|c|}{1.77 (MSME) } & \multicolumn{2}{|c|}{1.05 (MSME) } & \multicolumn{2}{|c|}{ Specified $<2.5 \%$} \\
\hline
\end{tabular}




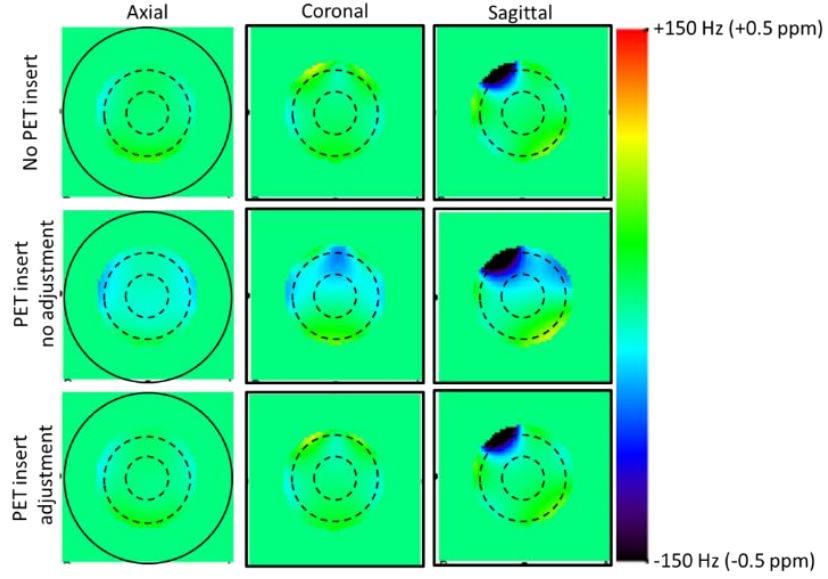

Figure 2. $B_{0}$ MRI field homogeneity maps acquired under three different conditions: Without the PET insert, with the PET insert but no adjustment of the center frequency nor shims and with the PET insert and pre-adjustment of the center frequency and shims performed. All maps are scaled from $150 \mathrm{~Hz}(-0.5 \mathrm{ppm})$ and $150 \mathrm{~Hz}(0.5 \mathrm{ppm})$. The dark cylindrical region of interest represents the PET field of view while the dashed spherical region of interest are the spherical volumes with diameter 20 and $40 \mathrm{~mm}$ used to derive the DSV20 and DSV40.

$\mathrm{Hz} /-0.96 \pm 5.16 \mathrm{~Hz}$ for DSV20 without and with the insert and $2.02 \pm 21.06 \mathrm{~Hz} /-0.98 \pm 20.45 \mathrm{~Hz}$ for DSV40). The acquisition of proton spectra using PRESS provided a linewidth of $6.7 / 17.1$ and $6.7 / 14.6 \mathrm{~Hz}$ (FWHM/FWTM) without and with the presence of the PET insert, respectively.

Figure 3 shows the flip angle homogeneity over the range of nominal flip angles investigated together with their normalized pulse amplitude. No major changes were observed in the presence of the PET insert (whatever the power conditions ON or OFF). The Quad40 coil had a well-preserved RF efficiency and preserved homogeneity in all conditions even for the lower flip angle. The Quad86 showed similar behavior but with an overall worst homogeneity and a degraded efficiency for low flip angles (normalized pulse magnitude of $0.78 \pm 0.18$ vs $0.92 \pm 0.04$ for the Quad40 for a nominal flip angle of 5 degrees and no PET insert in the FOV).

In terms of image quality, both Quad86 and Quad40 met the specifications in all conditions with a small decrease of the SNR. In particular, the maximum decrease was observed for the Quad86 using the short repetition time spin echo sequence with $-5.6 \%$. Homogeneity, asymmetry and image ghosting were all well within manufacturer specifications and no alteration of the RF transmission efficiency was observed as the preservation of the reference power attest (Table 1). EPI signal stability over a period of $10 \mathrm{~min}$ showed a deviation of 0.3 and $0.16 \%$ for the ROI peak to peak/mean without and with PET insert, which is well within the manufacturer specification of $<2 \%$. 


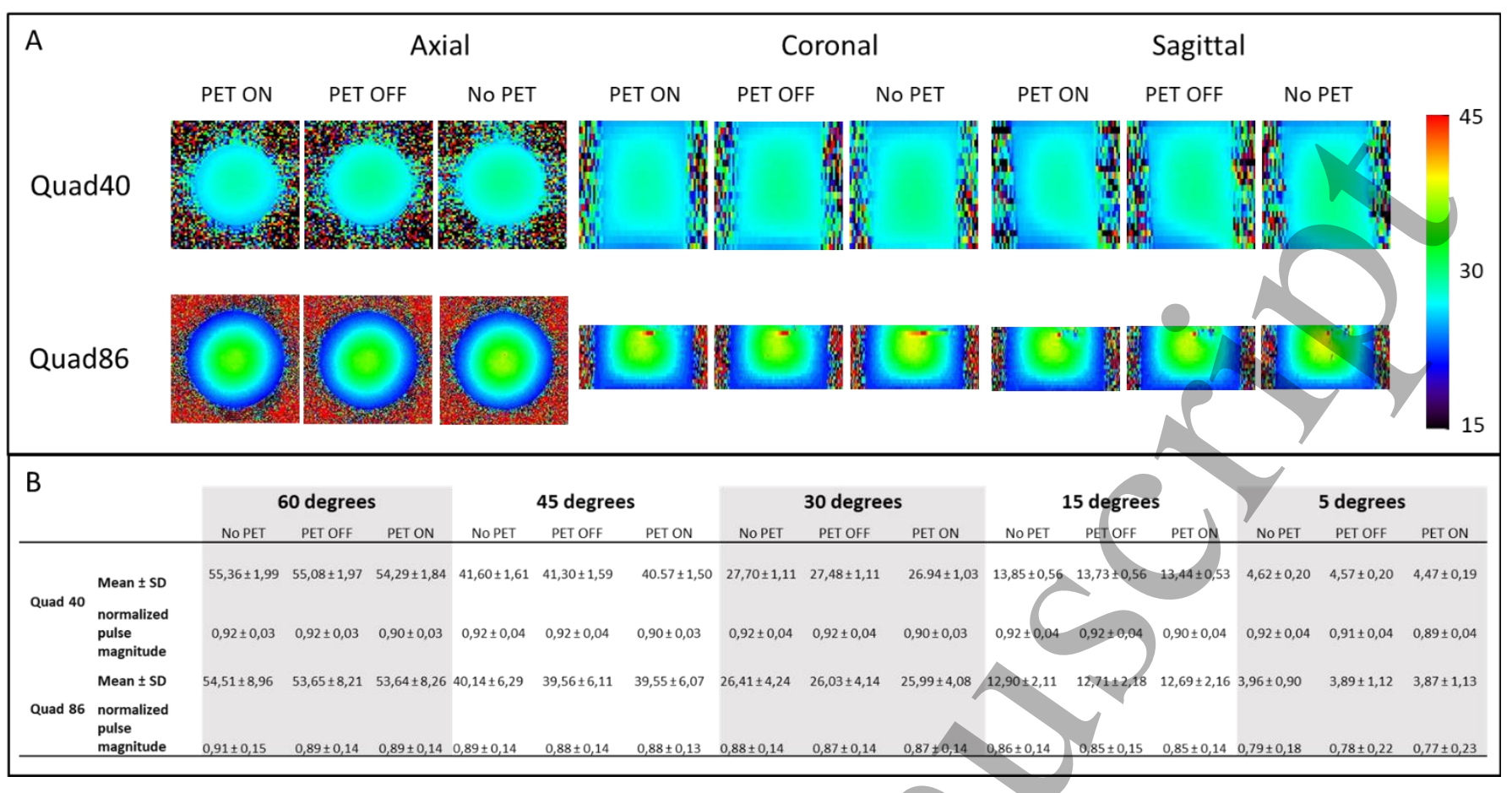

Figure 3. $B_{1}$ field maps for a nominal flip angle of 30 degrees under three different conditions (PET ON, PET OFF and No PET) and for the two main radiofrequency coils optimized for the simultaneous used with the PET insert (A). Radiofrequency homogeneity was assessed over cylindrical volumes of interest of 20 and $60 \mathrm{~mm}$ diameter for a length of $40 \mathrm{~mm}$ for the Quad40 and Quad86 respectively. Mean and standard deviation of the flip angle maps as well as the normalized pulse magnitude (voxel wise normalization by dividing with the nominal flip angle requested) are reported in $\mathrm{B}$.

\subsection{PET performance}

At the detector level (figure 4), the PET performance was as conditions (data not shown). No apparent distortions induced expected with no significant effect from any of the MRI by the MRI were observed, leading to a DOI FWHM around sequences (RARE, FLASH, EPI) investigated. The energy $1.6 \mathrm{~mm}$ in all conditions except for the FLASH sequence for resolution for the 3 tested modules and conditions (no MRI module 0 and 10, of 2.2 and $2.1 \mathrm{~mm}$, respectively. All spatial sequence, FLASH, RARE and EPI) was around $17 \%$ for the resolution measurements (FWHM and FWTM) are dataset with low activity. For modules 10 and 20 we observed a deterioration of around $10 \%$ of the energy resolution when summarized in figure 5 . 


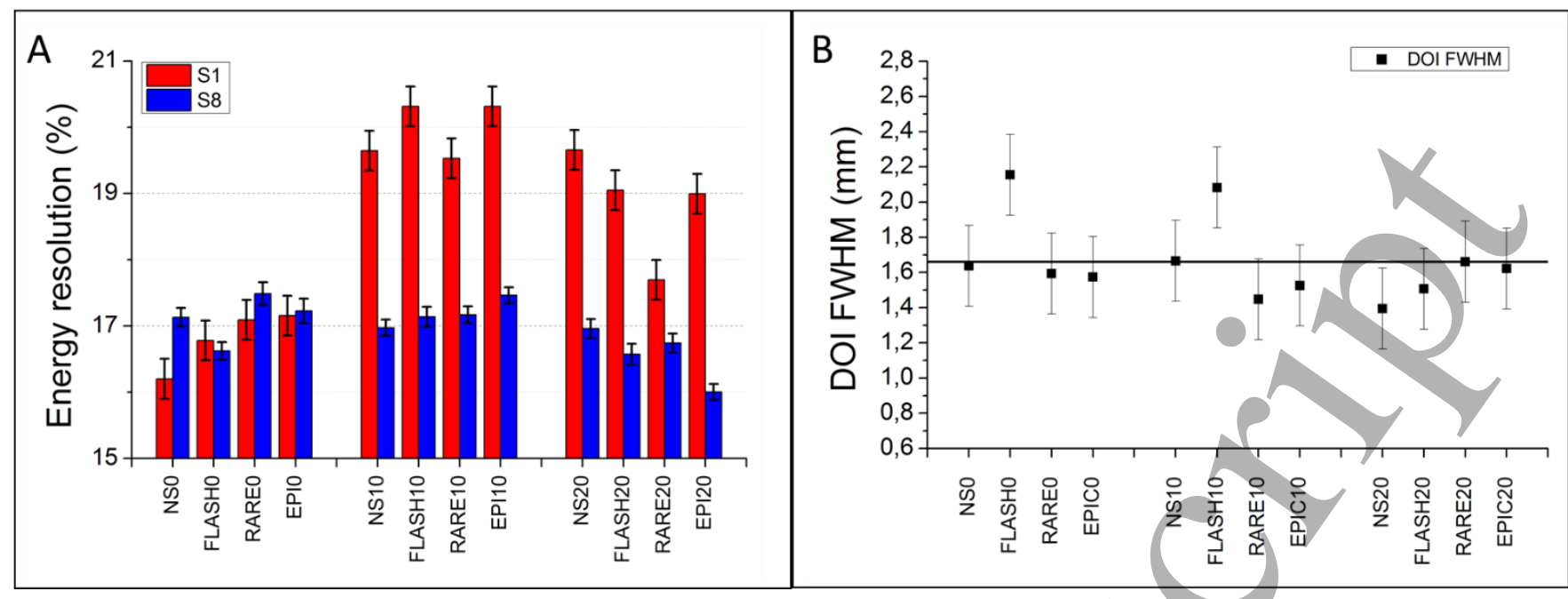

Figure 4. PET module performances. A: Effect of MRI (NS: no sequence, FLASH, RARE and EPI) energy resolution of PET detector modules 0,10 and 20 positioned at 0, 90, 180 degrees on the first, second and third ring respectively. S1 and S8 represent acquisition of homogenous phantom (cylinder of $30 \mathrm{~mm}$ diameter and $148 \mathrm{~mm}$ length centered in the PET FOV) with nominal activity of 44.3 and 1.1 MBq respectively. B: Depth of Interaction (DOI) resolution extracted from S1. 


\begin{tabular}{|c|c|c|c|c|c|c|c|c|c|c|c|c|c|}
\hline \multicolumn{14}{|c|}{ At axial center } \\
\hline \multirow{6}{*}{ No MRI } & & \multicolumn{2}{|c|}{$0 \mathrm{~mm}$} & \multicolumn{2}{|c|}{$5 \mathrm{~mm}$} & \multicolumn{2}{|c|}{$10 \mathrm{~mm}$} & \multicolumn{2}{|c|}{$15 \mathrm{~mm}$} & \multicolumn{2}{|c|}{$25 \mathrm{~mm}$} & \multicolumn{2}{|c|}{$35 \mathrm{~mm}$} \\
\hline & & FWHM & FWTM & FWHM & FWTM & FWHM & FWTM & FWHM & FWTM & FWHM & FWTM & FWHM & FWTM \\
\hline & Radial (X) & 0,68 & 1,24 & 0,87 & 1,60 & 0,96 & 1,75 & 0,92 & 1,68 & 0,96 & 1,74 & 1,06 & 1,93 \\
\hline & Tangential $(Y)$ & 0,86 & 1,56 & 0,78 & 1,43 & 0,85 & 1,54 & 0,84 & 1,54 & 0,97 & 1,76 & 0,86 & 1,57 \\
\hline & Axial (z) & 1,10 & 2,01 & 0,77 & 1,40 & 0,98 & 1,80 & 0,93 & 1,70 & 0,93 & 1,70 & 0,86 & 1,57 \\
\hline & Volume $\left(\mathrm{mm}^{3}\right)$ & 0.64 & & 0.52 & & 0.80 & & 0.72 & & 0.87 & & 0.78 & \\
\hline \multirow{4}{*}{ EPI } & Radial (x) & 0,63 & 1,14 & 0,88 & 1,61 & 0,90 & 1,65 & 0,93 & 1,69 & 0,93 & 1,69 & 1,02 & 1,87 \\
\hline & Tangential $(Y)$ & 0,87 & 1,59 & 0,79 & 1,43 & 0,86 & 1,56 & 0,85 & 1,54 & 0,98 & 1,79 & 0,89 & 1,62 \\
\hline & Axial (z) & 1,11 & 2,02 & 0,78 & 1,42 & 1,00 & 1,81 & 0,94 & 1,72 & 0,94 & 1,71 & 0,86 & 1,57 \\
\hline & Volume $\left(\mathrm{mm}^{3}\right)$ & 0.61 & & 0.54 & & 0.77 & & 0.74 & & 0.86 & & 0.78 & \\
\hline \multirow{4}{*}{ RARE } & Radial $(x)$ & 0,77 & 1,40 & 0,88 & 1,61 & 0,98 & 1,78 & 0,93 & 1,70 & 0,95 & 1,73 & 22 & 1,86 \\
\hline & Tangential $(Y)$ & 0,66 & 1,20 & 0,77 & 1,41 & 0,88 & 1,60 & 0,85 & 1,55 & 0,95 & 1,72 & 0.89 & 1,62 \\
\hline & Axial (z) & 0,80 & 1,46 & 0,77 & 1,41 & 0,99 & 1,80 & 0,96 & 1,75 & 0,91 & & 0,86 & 1,56 \\
\hline & Volume $\left(\mathrm{mm}^{3}\right)$ & 0.41 & & 0.52 & & 0.85 & & 0.76 & & 0.82 & & 78 & \\
\hline \multicolumn{14}{|c|}{ At $1 / 4$ axial FOV from center } \\
\hline & & \multicolumn{2}{|c|}{$0 \mathrm{~mm}$} & \multicolumn{2}{|c|}{$5 \mathrm{~mm}$} & \multicolumn{2}{|c|}{$10 \mathrm{~mm}$} & \multicolumn{2}{|c|}{$15 \mathrm{~mm}$} & \multicolumn{2}{|c|}{$25 \mathrm{~mm}$} & \multicolumn{2}{|c|}{$35 \mathrm{~mm}$} \\
\hline \multirow{5}{*}{ No MRI } & & FWHM & FWTM & FWHM & FWTM & FWHM & FWTM & FWHM & FWTM & FWHM & FWTM & FWHM & FWTM \\
\hline & Radial (X) & 0,75 & 1,38 & 0,90 & 1,63 & 0,94 & 1,72 & 0,92 & 1,68 & 0,97 & 1,76 & 0,94 & 1,7 \\
\hline & Tangential $(Y)$ & 0,80 & 1,46 & 0,81 & 1,48 & 0,84 & 1,54 & 0,85 & 1,55 & 1,05 & 1,90 & 0,89 & 1,62 \\
\hline & Axial (z) & 0,88 & 1,60 & 0,89 & 1,62 & 0,88 & 1,60 & 0,93 & 1,70 & 0,92 & 1,68 & 0,91 & 1,66 \\
\hline & Volume (mm) & 0.53 & & 0.65 & & 0.69 & & 0.73 & & & & 0.76 & \\
\hline \multirow{4}{*}{ EPI } & Radial (x) & 0,76 & 1,38 & 0,89 & 1,63 & 0,95 & 1,73 & 0,92 & 1,69 & 0,99 & 1,81 & 0,94 & 1,71 \\
\hline & Tangential $(Y)$ & 0,81 & 1,47 & 0,81 & 1,47 & 0,85 & 1,55 & 0,85 & 1,54 & 1,05 & 1,91 & 0,92 & 1,67 \\
\hline & Axial (z) & 0,88 & 1,61 & 0,90 & 1,64 & 0,91 & 1,65 & 0,94 & 1,72 & 0,95 & 1,73 & 0,87 & 1,59 \\
\hline & Volume $\left(\mathrm{mm}^{\prime}\right)$ & 0.54 & & 0.65 & & 0.73 & & & & 0.99 & & 0.75 & \\
\hline \multirow{4}{*}{ RARE } & Radial (x) & 0,71 & 1,29 & 0,89 & 1,63 & 0,94 & 1,54 & 0,93 & 1,70 & 0,97 & 1,78 & 0,95 & 1,74 \\
\hline & Tangential $(Y)$ & 0,77 & 1,41 & 0,81 & 1,47 & 0,85 & 1,55 & 0,85 & 1,55 & 1,05 & 1,92 & 0,93 & 1,70 \\
\hline & Axial (z) & 0,90 & 1,64 & 0,91 & 1,65 & 0,90 & 1,64 & 0,96 & 1,75 & 0,93 & 1,69 & 0,91 & 1,68 \\
\hline & Volume $\left(\mathrm{mm}^{3}\right)$ & 0.49 & & 0.66 & & 0.72 & & 0.76 & & 0.95 & & 0.80 & \\
\hline
\end{tabular}

\section{B}

C

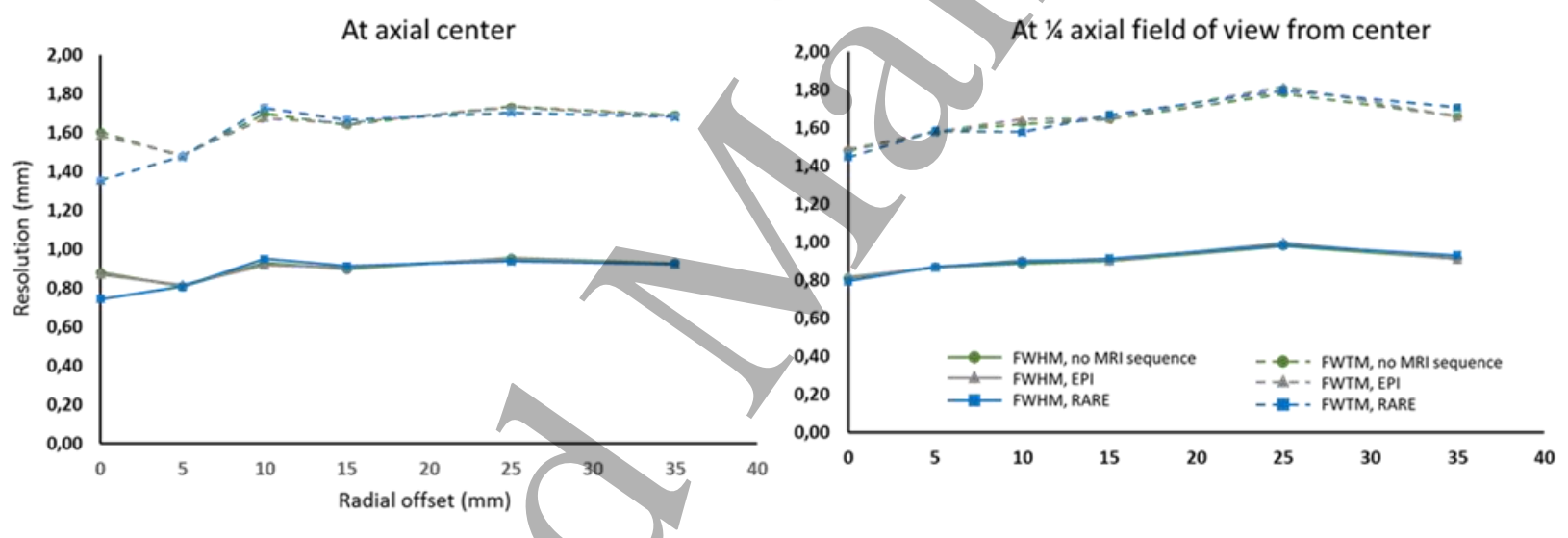

Figure 5. PET spatial resolution (FWHM and FWTM) measured in three orientations at the axial center of the FOV and at one fourth $(+37.5 \mathrm{~mm})$ of the axial FOV from the center of the axial FOV and at different radial offsets from the center (A). B and C: average spatial resolution (FWHM and FWTM) at the different location of the FOV. All acquisitions were performed inside the MRI without any MRI acquisition and with a RARE and EPI with at least $10^{6}$ coincidences (5 min acquisition time). Data reconstructed using MLEM algorithm with $0.25 \mathrm{~mm}$ voxel size and 25 iterations.

The average FWHM at the different positions in the FOV, including the very edge at $35 \mathrm{~mm}$ radial offset, were all below $1 \mathrm{~mm}$. EPI and RARE sequences had no effect on the resolution measurements. Overall, the volumetric resolution was ranging from 0.41 to $0.87 \mathrm{~mm}^{3}$ at the axial center and, 0.49 to $0.99 \mathrm{~mm}^{3}$ at a quarter of the axial center with a relatively well-preserved resolution at all radial offsets (figure $5 \mathrm{~B}$ and $\mathrm{C}$ ). This was confirmed by the acquisition of data using the Derenzo phantom (figure 6), in which rods as small as $0.7 \mathrm{~mm}$ can be resolved when scanning for 1 hour with an approximate activity of $30 \mathrm{MBq}$. Moreover, shorter acquisitions of 10-20 min with activities ranging from 13.3 to 5.6 MBq, and multiple MRI sequences (no MRI, RARE, FLASH, EPI and UTE) did not show major differences of image quality or resolution, as depicted by the profiles across the $0.9 \mathrm{~mm}$ rods. This was also observed for the small size source measurements. 

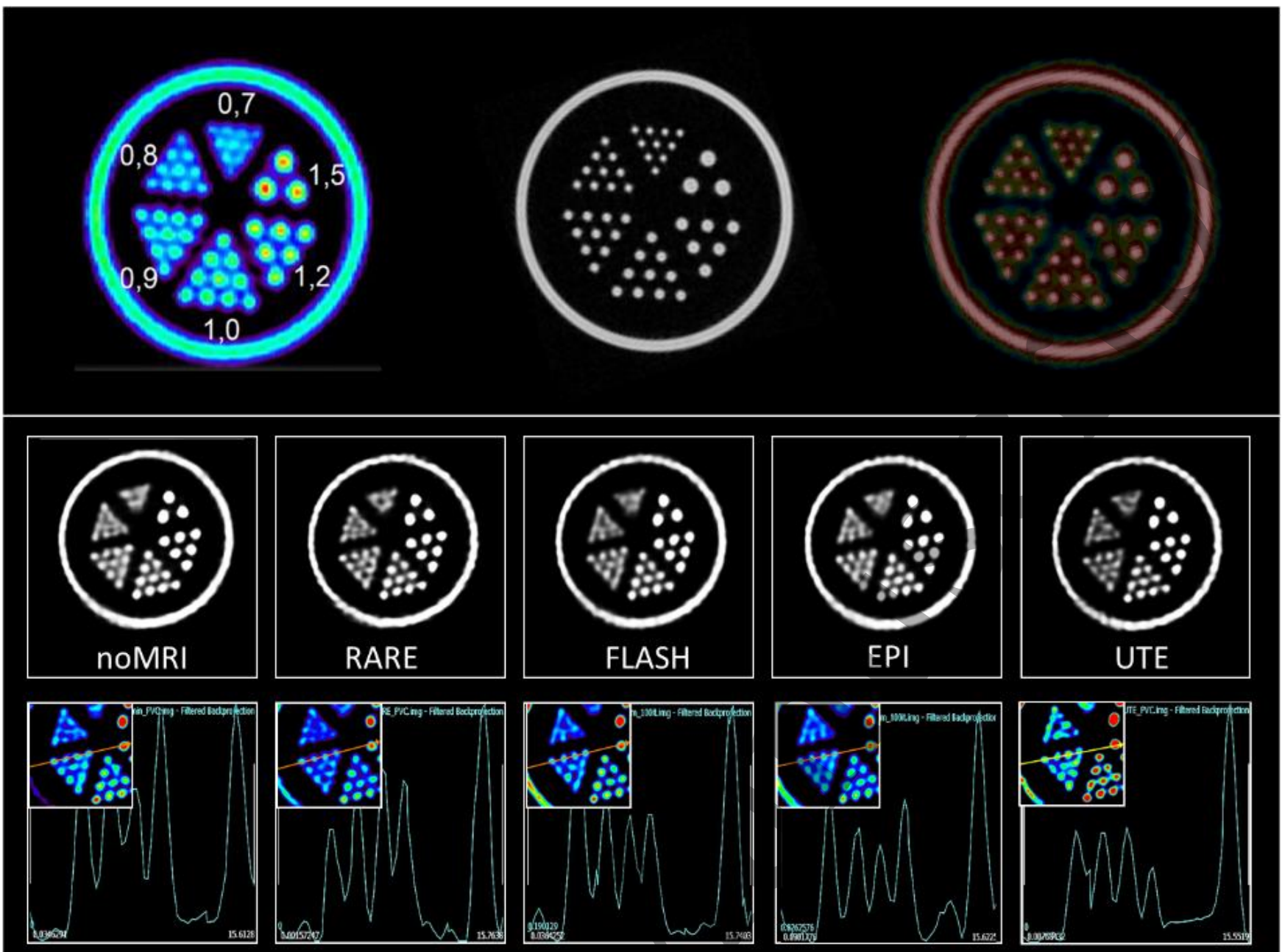

Figure 6. Derenzo Phantom images. Top row shows Derenzo imaged for 1 hour starting with $30 \mathrm{MBq}$ of $\left[{ }^{18} \mathrm{~F}\right] \mathrm{FDG}$ on the left, center MRI image (T2 weighted 3D-TurboRARE) and fusion on the right. Middle row shows the same phantom acquired for $20 \mathrm{~min}$ and loaded with activities ranging 10-5 MBq under different MRI sequence acquisitions. The bottom row shows the profiles of the $0.9 \mathrm{~mm}$ Derenzo capillaries in those MRI scenarios.

We determined a PET sensitivity of $11.0 \%$ at the center of the FOV, which progressively decreased to $6.6 \%$ at $40 \mathrm{~mm}$ axial offset (figure 7A), as expected.

The simultaneous MRI acquisition using RARE and EPI sequences did not alter the sensitivity profile, resulting in an absolute difference to the case without MRI sequence condition below $0.1 \%$ (figure $7 \mathrm{~B}$ ). Figure $7 \mathrm{C}$ and $\mathrm{D}$ report the count rates (random, scatter, total, true and NECR) performance using the mouse and rat phantoms. The peaks for true and noise equivalent count rates for the mouse phantom were $628 \mathrm{kcps}$ at $28 \mathrm{MBq}$ and $486 \mathrm{kcps}$ at $23 \mathrm{MBq}$. However, for the rat phantom we measured $410 \mathrm{kcps}$ at $30 \mathrm{MBq}$ and 239 kcps at $23 \mathrm{MBq}$. The acquisition of the image quality phantom also exhibited high recovery coefficients in all conditions, being for $3 \mathrm{~mm}$ rod of $0.91,0.94,0.89,0.91,0.93$ and 0.89 for 


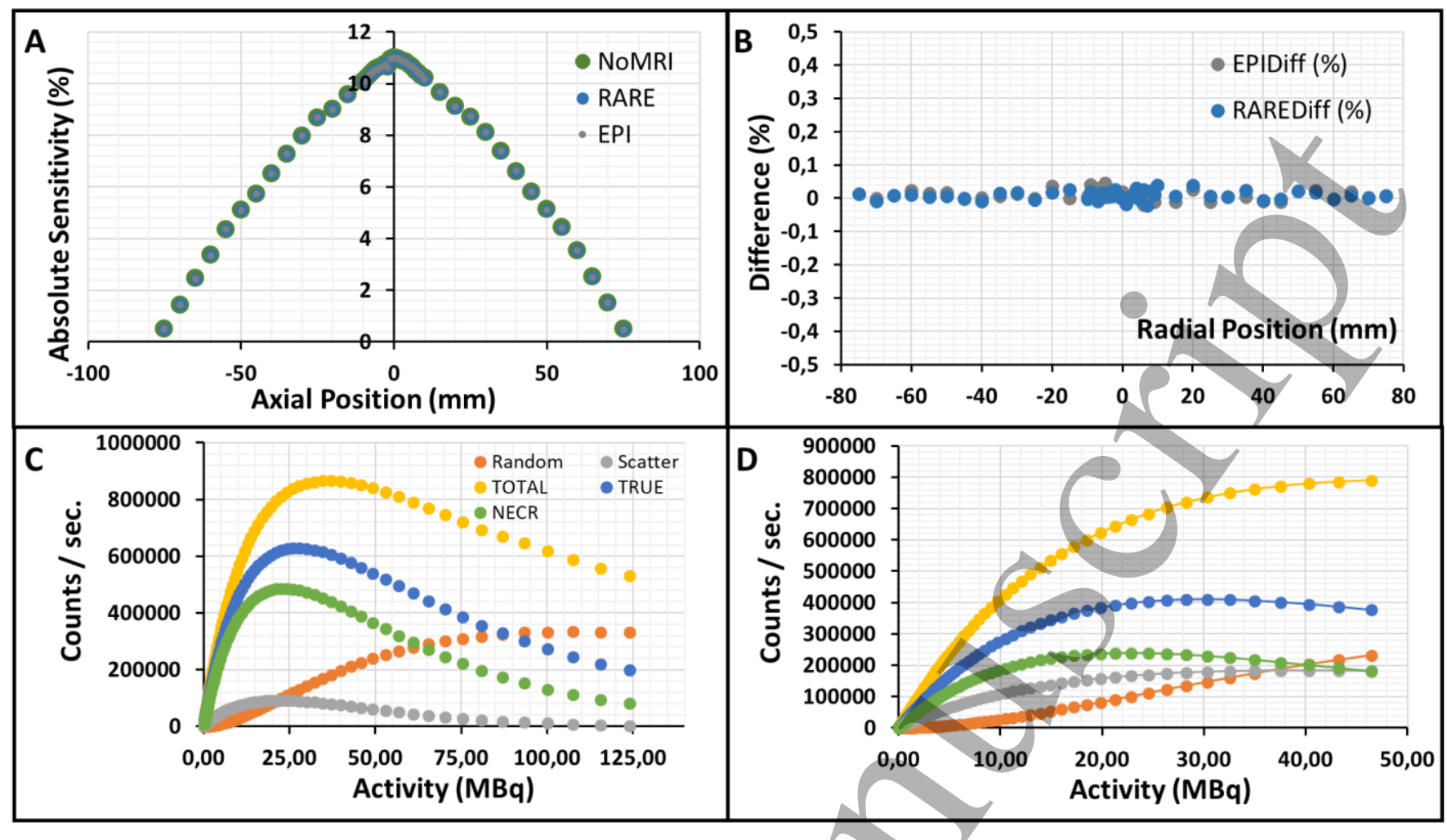

Figure 7. PET sensitivity, scatter fraction, count losses and random coincidence. A: PET absolute sensitivity profile with no MRI and with simultaneous acquisition with MRI (RARE and EPI) B: Sensitivity variation (\%) when MRI is simultaneously acquired using EPI and RARE sequences. C and D: Count rate performance using mouse and rat phantom respectively.

no MRI, EPI, FLASH, RARE, UTE and EPI high duty cycle $=0.9992)$. The average error in the calibration range $(1-32$ (table 3). We found a respective uniformity of 6.50,7.29,7.36, MBq) was $-1.17 \pm 1.48 \%,-1.17 \pm 1.52 \%,-0.89 \pm 1.64 \%$ $6.33,6.60$, and $6.65 \%$, and unaltered spill-over ratios of 0.12 and $-0.66 \pm 1.99 \%$ for without MRI, FLASH, RARE and EPI. for the air cavity and 0.21-0.23 for the water filled cavity. When using the no-sequence condition as a reference for the Finally, the system linearity was checked without MRI, linear regression and computing the residual from other FLASH, RARE and EPI sequences (figure 8). The regression conditions, we found absolute errors below $1 \%(-0.50 \pm 0.29$, coefficient showed a high degree of linearity preservation $\left(\mathrm{R}^{2} \quad-0.33 \pm 0.74\right.$ and $-0.33 \pm 0.17 \%$ for FLASH, RARE and EPI).

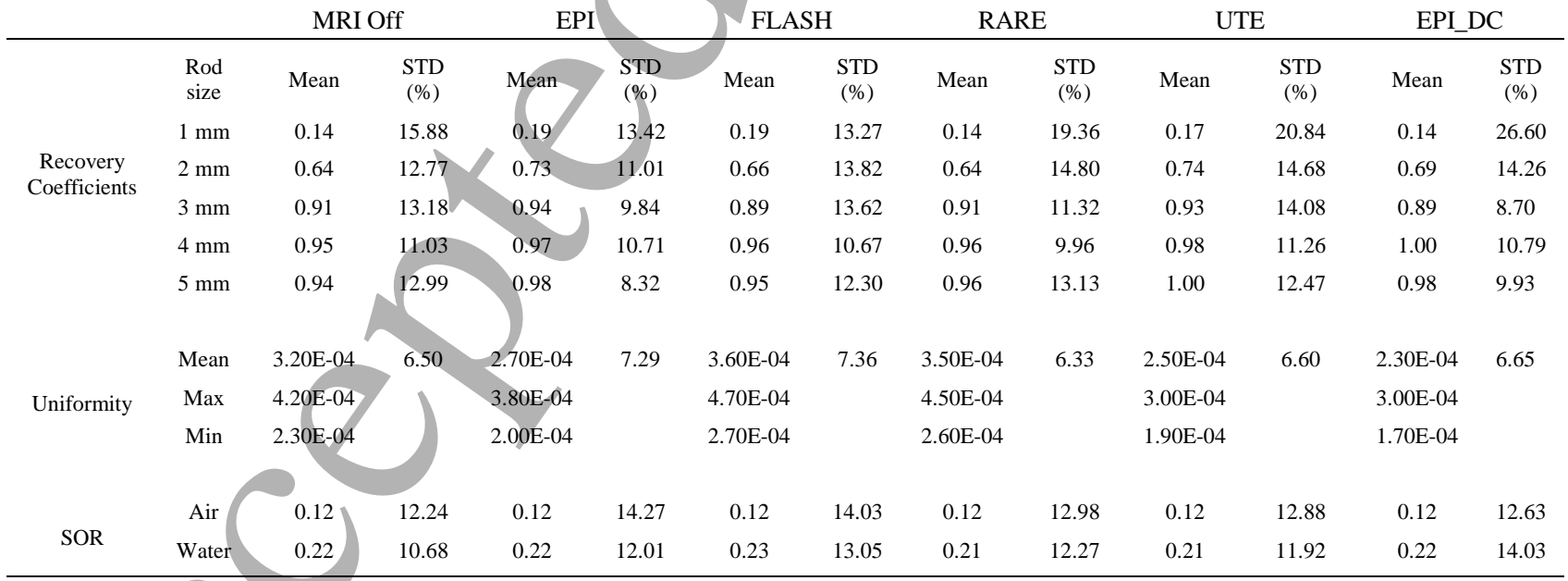

Table 3. PET image quality assessment. All data were reconstructed using MLEM reconstruction with $0.25 \mathrm{~mm}$ isotropic pixel size, 25 iterations and no partial volume correction. EPI: Echo Planar Imaging, FLASH: Fast low angle shot, RARE: 
Rapid acquisition with refocused echo, UTE: Ultrashort echo time, EPI_DC: EPI with high gradient duty cycle (75\%). SOR: Spill-over ratio.

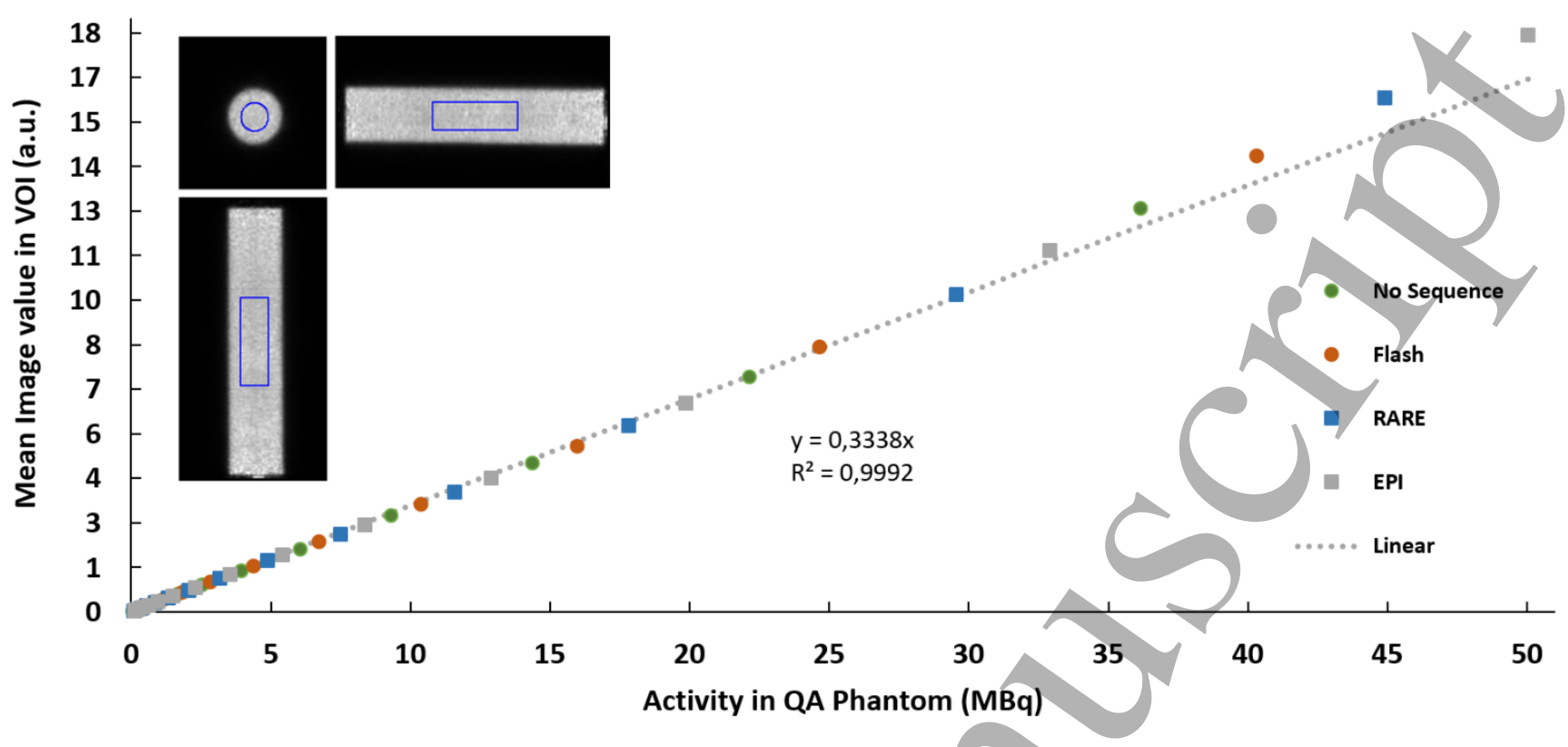

Figure 8. Linearity for quantitated PET Imaging. Phantom Size: $30 \mathrm{~mm}$ in diameter, $140 \mathrm{~mm}$ in length. Linear regression was performed using data points with an activity of 1 to $32 \mathrm{MBq}$. The average error in the calibration range (1-32MBq) was $-1.17 \pm 1.48 \%,-1.17 \pm 1.52 \%,-0.89 \pm 1.64 \%$ and $-0.66 \pm 1.99 \%$ for No Sequence, FLASH, RARE and EPI respectively. The average error for activities $\leq 50 \mathrm{MBq}$ was $-2.22 \pm 2.93 \%, 1.21 \pm 2.98 \%, 1.23 \pm 3.09 \%$ and $1.35 \pm 3.13 \%$ for No Sequence, FLASH, RARE and EPI respectively. The maximum error $(7.57 \%)$ was seen at $50 \mathrm{MBq}$, the highest activity tested. Using the linear regression from the control condition (No Sequence) provides an absolute error of $-0,50 \pm 0,29$, $0,33 \pm 0,74$ and $-0,33 \pm 0,17 \%$ for FLASH, RARE and EPI respectively.

\subsection{In-vivo imaging}

Figure 9 shows a sample of different PET/MRI in-vivo applications. Panel A represents an overlay of the rat brain $\left[{ }^{18} \mathrm{~F}\right] \mathrm{FDG}$ uptake and its corresponding anatomical MRI reference from which we can easily depict the increase $\left[{ }^{18} \mathrm{~F}\right]$ FDG uptake in the cortices and thalamic regions. Panel B and $\mathrm{C}$ are from the same dataset with $\mathrm{B}$ being the static reconstruction while $\mathrm{C}$ was reframed according to the ECG information to generate 8 cardiac frames. From B, one can appreciate the extended axial FOV of the PET insert $(150 \mathrm{~mm})$ enabling to scan an adult rat from nose to lower abdomen in a single bed position. From the maximum intensity projection
(MIP) of the static reconstruction we can appreciate the uptake distribution in the different organs brain, heart, Harderian glands and small organs such as adrenals. The cardiac reframing (C) showed a left ventricle uptake but also the right ventricle. The corresponding MRI showed a high contrast between the myocardial cavity (bright blood imaging) and the myocardial tissue with high resolution enabling to see the papillary muscles. Finally, from the whole-body MRI scan (D') of the mouse, one can easily identify all organs thanks to the high soft tissue contrast and spatial resolution of MRI. The corresponding MIP of the $\left[{ }^{18} \mathrm{~F}\right] \mathrm{NaF}$ scan (D',') demonstrates once again the good resolution of the PET insert with the rib cage and vertebral details resolved. 

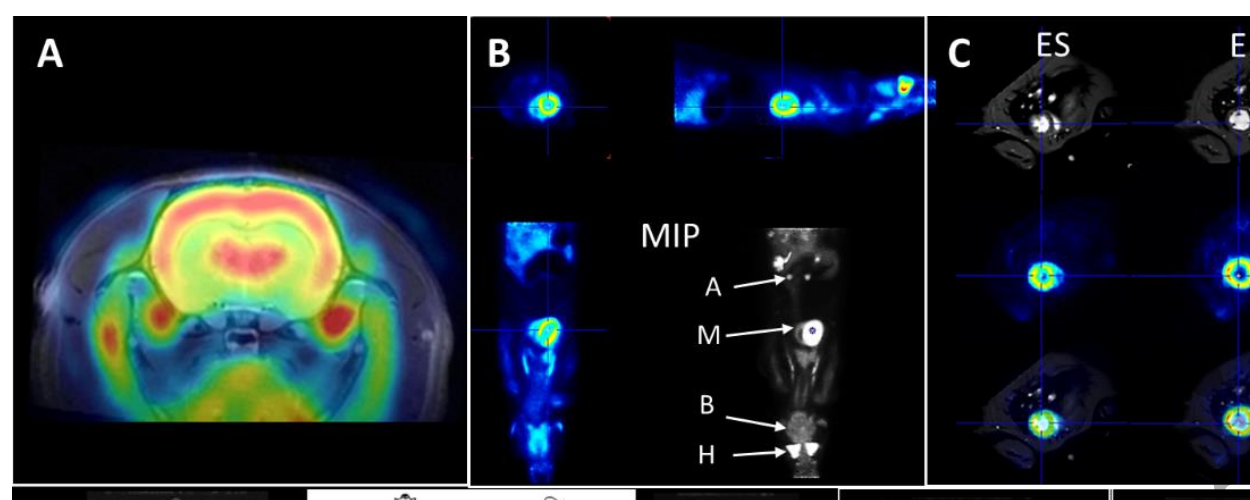

\section{ED}
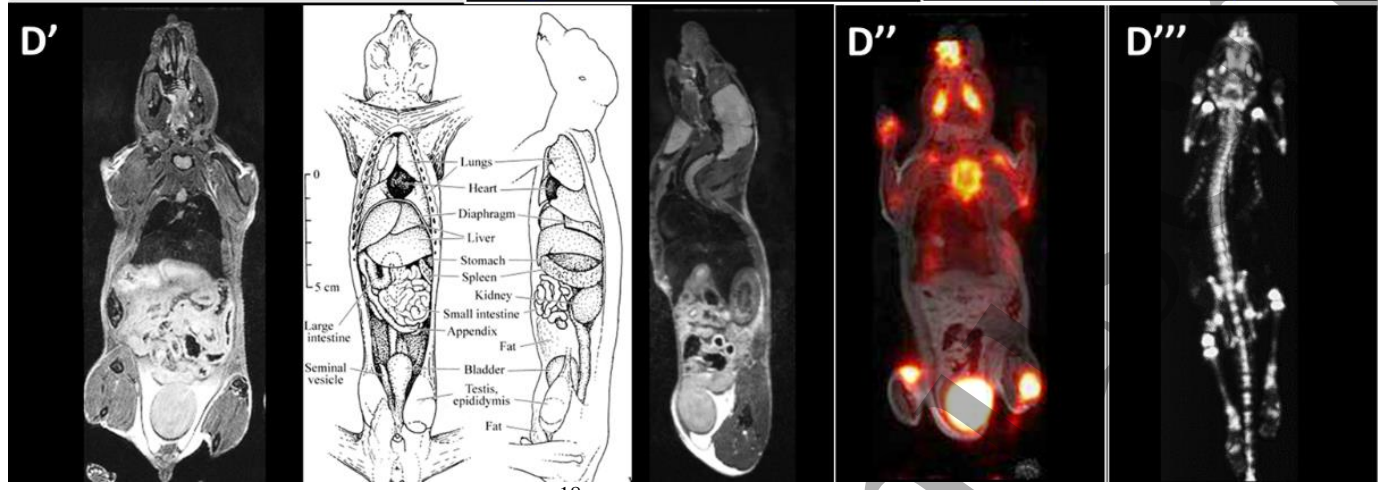

Figure 9. In-vivo PET/MRI in rodents. A: Rat brain $\left[{ }^{18} \mathrm{~F}\right] \mathrm{FDG}$ scan overlaid on 2D-TurboRARE anatomical scan. B: Ungated, single bed position rat $\left[{ }^{18} \mathrm{~F}\right] \mathrm{FDG}$ and its corresponding ECG-gated cardiac images (C) in end-diastole (ED) and end-systole (ES). Note the uptake from different organs from the static reconstruction (A: adrenals, M: myocardium, B: brain, H: harderian glands). D: Whole body mouse scan with a 3D-TurboRARE anatomical scan (D') in which organs are easily depicted. D', $\left[{ }^{18} \mathrm{~F}\right] \mathrm{NaF}$ Bone scan and $\mathrm{D}$ ', the overlay of the $\left[{ }^{18} \mathrm{~F}\right] \mathrm{NaF}$ PET' scan and the anatomical MRI reference.

\section{Discussion}

We hereby describe the full characterization of the Bruker preclinical SiPM PET insert in terms of the PET performance but also its potential interference with the MRI and vice-versa. The 3-ring PET insert outlined here offers one of the largest FOV for rodent imaging (Cal-Gonzalez et al. 2018) with 80 $\mathrm{mm}$ diameter and an axial extend of $150 \mathrm{~mm}$, enabling simultaneous PET/MRI scanning of whole-body mouse or covering rat body from nose to lower abdomen in a single bed position. It is therefore designed not only to image specific organs but also to offer the capability to investigate new tracer whole-body biodistribution or dynamic scanning with kinetic modeling using image-based arterial input function since the heart and big blood vessels are always within the FOV, even when performing brain or xenograft scans. From the MRI point of view, there is a lack of standard protocols across platforms and manufacturers enabling the evaluation and comparison of the different preclinical MRI systems (Nagy et al. 2013). We therefore adapted the MRI acceptance protocol from the manufacturer in order to capture performance criteria such as RF noise, $\mathrm{B}_{0}$ and $\mathrm{B}_{1}$ field homogeneities, $\mathrm{SNR}$, image homogeneity, ghosting, and signal stability over time. Only minor effects related to the presence of the PET insert have been observed. The presence of the PET insert did not induce added radiofrequency noise as depicted in figure 1 . The design of the PET insert is such that all power supplies and data acquisition electronics are within a shielded cabinet placed at the back of the magnet. While this may still be a problem for clinical scanners with a much wider bore size (Lee et al. 2018), the shielding here was sufficient to not have radiofrequency leakage from the cabinet as previously described for other preclinical PET insert design (Wehrl et al., 2011). This also reduced the potentially induced RF noise since all digital signal processing are performed outside the MRI bore. Within the insert, all cables and detector modules are also RF shielded. This last point does not affect the performance of the RF transmission since the RF coil in this set up is placed inside the PET insert as opposed to clinical systems using RF coils around the PET ring for signal transmission (Kang et al., 2015, Lee et al. 2018). RF noise added by the presence of the PET insert would also have been noticed from our SNR measurements and potential ghosting (table 1). The second potential main problem when combining PET and MRI is the distortion of the main magnetic field $\left(\mathrm{B}_{0}\right)$. From our $\mathrm{B}_{0}$ map measurements we did see a small change of the global (around $20 \mathrm{~Hz}$ ) and local field homogeneities (increase of $10 \mathrm{~Hz}$ of deviation from the DSV 40). However, this was well compensated by the automatic re-adjustment of the center frequency and shims. Again, major alterations of $B_{0}$ homogeneity would have drastically altered the quality of the gradient echo sequences such as FLASH and EPI. The performances of two quadrature birdcage coils (Quad40 and Quad86) were also well preserved and not degraded by the 
presence of the PET insert. Both coils' matching and tuning were preserved in all conditions providing an efficient transmission of the radiofrequency pulses with low power (Omidvari et al. 2018). The design of having the RF coils in close vicinity of the subject (inside the PET insert) as opposed to some configurations in clinical scanners (Kang et al. 2015, Lee et al. 2018) offer the advantage to alleviate potential reduction of the RF efficiency and effect on the $B_{1}$ homogeneity.

Finally, all together the evaluation of the SNR, signal homogeneity and ghosting demonstrate no drastic deterioration of the image quality. PET detectors worked as expected and reported from previous work (Gonzalez et al. 2016). Overall our assessment showed a slight alteration of the energy resolution when using very high activities (35.2-44.3 $\mathrm{MBq}$ ) as opposed to data acquired with activity around $1 \mathrm{MBq}$ (average energy resolution of $18.5 \pm 1.5 \%$ vs $16.6 \pm 0.4 \%$ for high and low activity conditions respectively) probably due to pile-up effect. FLASH, RARE and EPI do not have significant effect on the energy resolution in all three modules tested and independently of the activity in the FOV. For instance, module 10 exhibited energy resolutions of $17.1 \pm 0.1,17.2 \pm 0.1,17.5$ \pm 0.1 and $17.0 \pm 0.1 \%$ for FLASH, RARE, EPI, and no MRI sequence, respectively. These data demonstrate the robustness and reproducibility of the quantitative PET values when MRI is operated simultaneously. This was also confirmed by acquiring a PET linearity test (figure 8 ) with a maximum error in the calibration range $(1-32 \mathrm{MBq})$ induced by the FLASH sequence being less than $1.17 \%$ (less than the error induced by the approximation in evaluating the animal injected activity or the inter-scan variability due to PET count statistics and homogeneity). The accuracy in the impact position was first tested at the detector level by estimating the DOI resolution. The DOI resolution was found to have a FWHM around 1.6 $\mathrm{mm}$ with no major effect induced by MRI. The acquisition of the Na-22 point source data according to the NEMA protocol, showed a sub-millimeter resolution (average FWHM of 0.83 $\mathrm{mm}$ ). Because of the DOI correction implemented as single side reading in a monolithic scintillator, the insert is still compact in size with crystal thickness of $10 \mathrm{~mm}$ as compared to other systems using dual-layer crystal (Cal-Gonzalez et al. 2018) providing thus less space to fit the radiofrequency coil and the animal bed. As already reported, the use of continuous crystals and the capability of mapping the scintillation light distribution, allows one to reach accurate DOI information and, thus, correcting for the parallax error. This results in a homogenous spatial resolution across the entire FOV, with the same average FWHM of $0.92 \mathrm{~mm}$ at the axial center and at $37.5 \mathrm{~mm}$ axial offset and at $35 \mathrm{~mm}$ radial offset (right at the edge of the FOV of the PET insert). This added feature becomes very appreciable for scanning simultaneously multiple animals while preserving the spatial resolution in the whole FOV (Habte et al. 2013). Moreover, none of the MRI sequences tested showed any degradation of the PET image resolution. While using small size $\mathrm{Na}-22$ sources, it remains difficult to appreciate if resolution could be improved by reduction of the positron range within a strong magnetic field
(Huang et al. 2014) but this merits further investigations with radionuclides with higher positron energy such as oxygen-15, zirconium- 89 and gallium- 68 . The second advantage of using a large axial FOV design with the use of monolithic crystals coupled to SiPM resides in the high sensitivity $(11 \%)$ and count rates, offering low dose imaging capability as already published using a similar design integrated with $\mathrm{CT}$ (Molinos et al. 2019). All together we report here that PET image quality is not affected by the magnetic field nor the radiofrequency and gradient field using most of the conventional MRI sequences used in-vivo. This statement holds with even EPI high duty cycles which are often reported as the most problematic due to the fast gradient switching and gradient strength needed (Stortz et al. 2018). The image quality criteria such as recovery coefficients, uniformity and spill-over ratios were all comparable to similar PET-insert design from other manufacturer or academic groups or conventional preclinical PET system (Cal-Gonzalez et al. 2018). This could also be appreciated from the different invivo examples reported in this study with exquisite resolution enabling to resolve rat brain cortices in a single animal or even the rat right myocardium.

\section{Acknowledgements}

We acknowledge the KU Leuven core facility, Molecular Small Animal Imaging Center (MoSAIC), for their support with obtaining scientific data presented in this paper. This work was supported by Stichting tegen Kanker (2015-145, Christophe M. Deroose) and Hercules foundation (AKUL/13/029, Uwe Himmelreich) for the purchase of the PET and MRI equipment respectively. The work was supported by the following funding organizations: European Commission for the PANA project (H2020-NMP-2015-twostage, grant 686009) and the European ERA-NET project 'CryptoView' (3rd call of the FP7 program Infect-ERA).

\section{References}

1. Balezeau F, Eliat PA, Cayamo AB, et al. Mapping of low flip angles in magnetic resonance. Phys Med Biol. 2011 Oct 21;56(20):6635-47.

2. Benlloch JM, González AJ, Pani R, et al.. The MINDVIEW project: First results. Eur Psychiatry. 2018 Apr;50:21-27.

3. Cal-Gonzalez J, Rausch I, Shiyam Sundar LK, et al. Hybrid imaging: Instrumentation and data processing. 2018 Front. Phys. 6: 47.

4. Catana C, Wu Y, Judenhofer MS, Qi J, Pichler BJ, Cherry SR. Simultaneous acquisition of multislice PET and MR images: initial results with a MRcompatible PET scanner. J Nucl Med. 2006 Dec;47(12):1968-76.

5. Clark DP, Badea CT. Micro-CT of rodents: stateof-the-art and future perspectives. Phys. Med. 2014 Sep; 30(6): 619-634. 
6. Drzezga A, Souvatzoglou M, Eiber M, et al.. First clinical experience with integrated whole-body PET/MR: comparison to PET/CT in patients with oncologic diagnoses. J Nucl Med. 2012 Jun;53(6):845-55.

7. González AJ, Aguilar A, Conde P, et al. A PET Design Based on SiPM and Monolithic LYSO Crystals: Performance Evaluation. IEEE Trans Nucl Sci 2016 Oct; 63(5): 2471-2477.

8. Gonzalez AJ, Gonzalez-Montoro A, Vidal LF, et al. Initial Results of the MINDView PET Insert Inside the 3T mMR. IEEE Trans Rad Plas Med Sci 2019 May; 3(3): 343-351.

9. Grant AM, Lee BJ, Chang CM, Levin CS. Simultaneous PET/MR imaging with a radio frequency-penetrable PET insert. Med Phys. 2017 Jan;44(1):112-120.

10. Habte F., Ren G, Doyle T.C., Liu H., Cheng Z., Paik D.S. Impact of a Multiple Mice Holder on Quantitation of High-Throughput MicroPET Imaging With and Without $\mathrm{Ct}$ Attenuation Correction. Mol Imaging Biol. 2013 Oct; 15(5): 569-575.

11. Hammer BE, Christensen NL, Heil BG. Use of a magnetic field to increase the spatial resolution of positron emission tomography. Med Phys. 1994 Dec;21(12):1917-20.

12. Huang S. Savic D., Yang J., Shreshta U, Seo Y. The Effect of Magnetic Field on Positron Range and Spatial Resolution in an Integrated WholeBody Time-Of-Flight PET/MRI System. IEEE Nucl Sci Symp Conf Rec (1997). 2014 Nov; 2014.

13. Jadvar H, Colletti PM. Competitive advantage of PET/MRI. Eur J Radiol. 2014 Jan;83(1):84-94.

14. Judenhofer MS, Catana C, Swann BK, et al. PET/MR images acquired with a compact MRcompatible PET detector in a 7-T magnet. Radiology. 2007 Sep;244(3):807-14.

15. Kang HG, Hong SJ, Ko GB, et al. Assessment of MR-compatibility of SiPM PET insert using short optical fiber bundles for small animal research. JINST. 2015 Dec, 10: P12008.

16. Kinahan PE, Townsend DW, Beyer T, Sashin D. Attenuation correction for a combined 3D PET/CT scanner.Med Phys 1998;25:2046-2053.

17. Ko GB, Yoon HS, Kim KY, et al. Simultaneous Multiparametric PET/MRI with Silicon Photomultiplier PET and Ultra-High-Field MRI for Small-Animal Imaging. J Nucl Med. 2016 Aug;57(8):1309-15.

18. Lee BJ, Grant AM, Chang CM, Watkins RD, Glover GH, Levin CS. MR Performance in the Presence of a Radio Frequency-Penetrable Positron Emission Tomography (PET) Insert for Simultaneous PET/MRI. IEEE Trans Med Imaging. 2018 Sep;37(9):2060-2069.
19. Loening AM, Gambhir SS. AMIDE: A Free Software Tool for Multimodality Medical Image Analysis. Molecular Imaging, 2(3):131-137, 2003.

20. Mannheim JG, Schmid AM, Schwenck J, et al. PET/MRI Hybrid Systems. Semin Nucl Med. 2018 Jul;48(4):332-347.

21. Maramraju SH, Smith SD, Junnarkar SS, et al. Small animal simultaneous PET/MRI: initial experiences in a 9.4 T microMRI. Phys Med Biol. 2011 Apr 21;56(8):2459-80.

22. Molinos C, Sasser T, Salmon P, et al. Low-Dose Imaging in a New Preclinical Total-Body PET/CT Scanner. Front Med (Lausanne). 2019 May 3;6:88.

23. Nagy K, Toth M, Major P, et al. Performance evaluation of the small-animal nanoscan PET/MRI system. J Nucl Med. 2013 Oct;54(10):1825-32.

24. Nanni C, Torigian DA. Applications of small animal imaging with PET, PET/CT, and PET/MR imaging. PET Clin. 2008 Jul;3(3):243-50.

25. Omidvari N, Cabello J, Topping G, et al. PET performance evaluation of MADPET4: a small animal PET insert for a 7 T MRI scanner. Phys Med Biol. 2017 Nov 1;62(22):8671-8692.

26. Omidvari N, Topping G, Cabello J, et al. MRcompatibility assessment of MADPET4: a study of interferences between an SiPM-based PET insert and a 7T MRI system. Phys Med Biol. 2018 May 1;63(9): 095002.

27. Pichler BJ, Judenhofer MS, Catana C, et al. Performance test of an LSO-APD detector in a 7-T MRI scanner for simultaneous PET/MRI. J Nucl Med. 2006 Apr;47(4):639-47.

28. Raylman RR, Majewski S, Lemieux SK, et al. Simultaneous MRI and PET imaging of a rat brain. Phys Med Biol. 2006 Dec 21;51(24):6371-9.

29. Roncali E, Cherry SR. Application of silicon photomultipliers to positron emission tomography. Ann Biomed Eng. 2011 Apr;39(4):1358-77.

30. Schug D, Lerche C, Weissler B, et al. Initial PET performance evaluation of a preclinical insert for PET/MRI with digital SiPM technology. Phys Med Biol. 2016 Apr 7;61(7):2851-78.

31. Shao Y, Cherry SR, Farahani K, et al. Simultaneous PET and MR imaging. Phys Med Biol. 1997 Oct;42(10):1965-70.

32. Steinert HC, von Schulthess GK. Initial clinical experience using a new integrated in-line PET/CT system. Br. J. Radiol 2002; 73: S36-38.

33. Stortz G, Thiessen JD, Bishop D, et al. Performance of a PET Insert for High-Resolution Small-Animal PET/MRI at 7 Tesla. J Nucl Med. 2018 Mar;59(3):536-542.

34. Townsend DW. Combined PET/CT: the historical perspective. Semin Ultrasound CT MR. 2008 August ; 29(4): 232-235. 
35. Vandenberghe S, Marsden PK. PET-MRI: a review of challenges and solutions in the development of integrated multimodality imaging. Phys Med Biol. 2015 Feb 21;60(4):R115-54.

36. Vaquero JJ, Kinahan P. Positron Emission Tomography: Current Challenges and Opportunities for Technological Advances in Clinical and Preclinical Imaging Systems. Annu Rev Biomed Eng. 2015;17:385-414.

37. von Schulthess GK, Schlemmer HP. A look ahead: PET/MR versus PET/CT. Eur J Nucl Med Mol Imaging. 2009 Mar;36 Suppl 1:S3-9.

38. Wehner J, Weissler B, Dueppenbecker PM, et al. MR-compatibility assessment oft he first preclinical PET-MRI insert equipped with digital silicon photomultipliers. Phys Med Biol. 2015 Mar 21;60(6):2231-2255.

39. Wehrl HF, Judenhofer MS, Thielscher A, et al. Assessment of MR compatibility of a PET insert developed for simultaneous multi-parametric PET/MR imaging on animal system operating at 7T. Magn. Reson. Med. 2011 Jan;65(1):269-279.

40. Yamamoto S, Imaizumi M, Kanai Y, et al. Design and performance from an integrated PET/MRI system for small animals. Ann Nucl Med. 2010 Feb;24(2):89-98.

41. Yamamoto S, Watabe T, Watabe H, et al. Simultaneous imaging using Si-PM-based PET and MRI for development of an integrated PET/MRI system. Phys Med Biol. 2012 Jan 21;57(2):N1-13.

42. Zaidi H, Montandon ML, Alavi A. The Clinical Role of Fusion Imaging Using PET, CT, and MR Imaging. PET Clin. 2008 Jul;3(3):275-91. 